Bulletin of the Seismological Society of America. Vol. 58, No. 2, pp. 689-709. April, 1968

\title{
PARKFIELD EARTHQUAKE OF JUNE 28, 1966: MAGNITUDE AND SOURCE MECHANISM
}

\author{
By Francis T. $\mathrm{Wu}_{\mathrm{U}}$
}

\section{ABSTRACT}

The Parkfield earthquake of June 28, 1966 (04:26:12.4 GMT) is studied using short-period and long-period teleseismic records. It is found that (1) $M_{b}=5.8$ and $M_{s}=6.4$ as compared to $M_{b}=5.4$ and $M_{s}=5.4$ for the foreshock 104:08:54), (2) both the Rayleigh and Love wave radiation patterns conform to those of a double couple at a depth of about $8.6 \mathrm{~km},(3)$ the main shock can be represented by a series of shocks separated in space and time.

The near-field strong-motion data support the last conclusion. Based on strong-motion seismograms, and the surficial evidences of the dimensions of the fault, the energy is found to be $10^{21}$ ergs.

\section{INTRODUCTION}

The Parkfield earthquake of 28 June 1966 has aroused extensive interest because of its location on the San Andreas fault where every breath has been monitored. The recordings of this event fall into three main categories: strong-motion seismometer and seismoscope records in the epicentral region; short-period seismometer records from Berkeley, Caltech and USCGS network (within an epicentral distance of $200 \mathrm{~km}$ ); and long period WWNSS and Canadian net records. In the first category we can procure information about the amplitude period and orbital motion of waves in the source region. This is the first time in seismological history such information was gathered systematically with an array within the source region. However, the mission of this instrument cluster being non-seismological in its design, no absolute or relative time was available on the records; it is impossible to decipher the nature of the waves recorded. In the second category, the seismograms are used to determine accurately the origin time and the epicenter, and also the first motions. The last category is the teleseismic records on long-period instruments $\left(t_{0}=15 \mathrm{sec}, t g=30 \mathrm{sec}\right)$; the body waves that were so predominant on the second category records now appear only as a faint trace in most cases. These last category records are the chief source of data for this study.

It must be noted that while the near-source waves must bear some relation to the far-field or teleseismic waves, the manner in which the orbital motions of the nearsource wave behave and the magnitude of the strain involved in these waves throw some doubt on a direct relationship; namely, the near-source waves may be nonlinear in the sense that they diminish much faster than linearly diminished body waves, and/or the material behaved non-linearly under the high stress. In either case the waves would have suffered a very severe attenuation in the immediate region close to the source; to recover the information contained in these waves from teleseismic waves well described by first-order linear elasticity theory, would be difficult if not impossible.

In using the teleseismic signals for source mechanism studies we are looking for 
average features of the earthquake processes such as the average velocity propagation of the source, the average length of the fault and so on.

\section{Magnitudes of the Foreshock and the Main Shock}

Richter magnitude scale as originally designed by Richter (1935) employed the maximum trace of short-period waves recorded on Wood-Anderson torsional seismometers to study the statistical characteristics of local earthquakes. Later, Gutenberg and Richter (1936) extended the same idea to categorize teleseisms based on the body waves $P, S, P P$ and the more prominent phase on a teleseismic record, the "20 second" surface waves. A relation between these two magnitude scales was subsequently sought to equalize them to yield the same result (Gutenberg and Richter, 1956). A basic assumption involved here is that the source spectrum for an earthquake of certain size is fixed in shape; thereby, knowing the amplitude at one frequency would suffice to calibrate the earthquake. In the process of developing the relation, a large number of stations and earthquakes were used; the relation yields rather consistent results for "average" earthquakes. The main shock of the Parkfield event is, however, a rather outstanding exception.

The fundamental formula used in calculation of magnitude $M$ based on surfacewave amplitude is

$$
M_{S}=\log _{10} A_{z}-\log _{10} B+\bar{\delta}+M_{R}+\eta\left(7.1-M_{c}\right)
$$

This formula, a modification (Bath, 1952) of Gutenberg and Richter's original formula, is for exclusive application with vertical component Rayleigh waves, where

$$
\begin{aligned}
A= & \text { the amplitude (in microns) of } 20 \text { second Rayleigh } \\
& \text { waves recorded on the vertical component of long- } \\
& \text { period seismometers, } \\
\log _{10} B= & \text { distance correction factor, } \\
\bar{\delta}+M_{R}= & \text { correction for depth, path, radiation pattern, etc., } \\
\eta= & 0.2 \text { for normal shock, } \\
M_{c}= & \text { the sum of the first four terms in the formula. }
\end{aligned}
$$

In the present investigation $\bar{\delta}+M_{R}$ is set to 0 , since we used a number of stations, and the radiation pattern is actually worked out.

For body waves we use the formula

$$
M_{b}=\log w / T+Q
$$

where

$$
\begin{aligned}
& w=\text { amplitude of the vertical component } P \text { wave } \\
& T=\text { period of the wave } \\
& Q=\text { equalizing factor (Gutenberg and Richter, 1956, Figure 5). }
\end{aligned}
$$

In this study body wave amplitudes and surface wave amplitudes are measured on WWNSS short-period records and long-period records, respectively. 
Table 1 shows the results obtained at various stations for the main shock and, for the purpose of comparison, the computed body-wave and surface-wave magnitudes for the foreshock at 04:08:54 approximately 17 minutes before the main shock.

The surface wave magnitudes for the main shock, computed from the available records, clearly show an azimuthal effect that is related mainly to the non-spherical symmetry of the source. For example, at College, Alaska (COL in Table I), the computed magnitude is evidently lower than at other stations. The severe attenuation of the 20 second waves while travelling across crustal boundaries and along the

TABLE 1

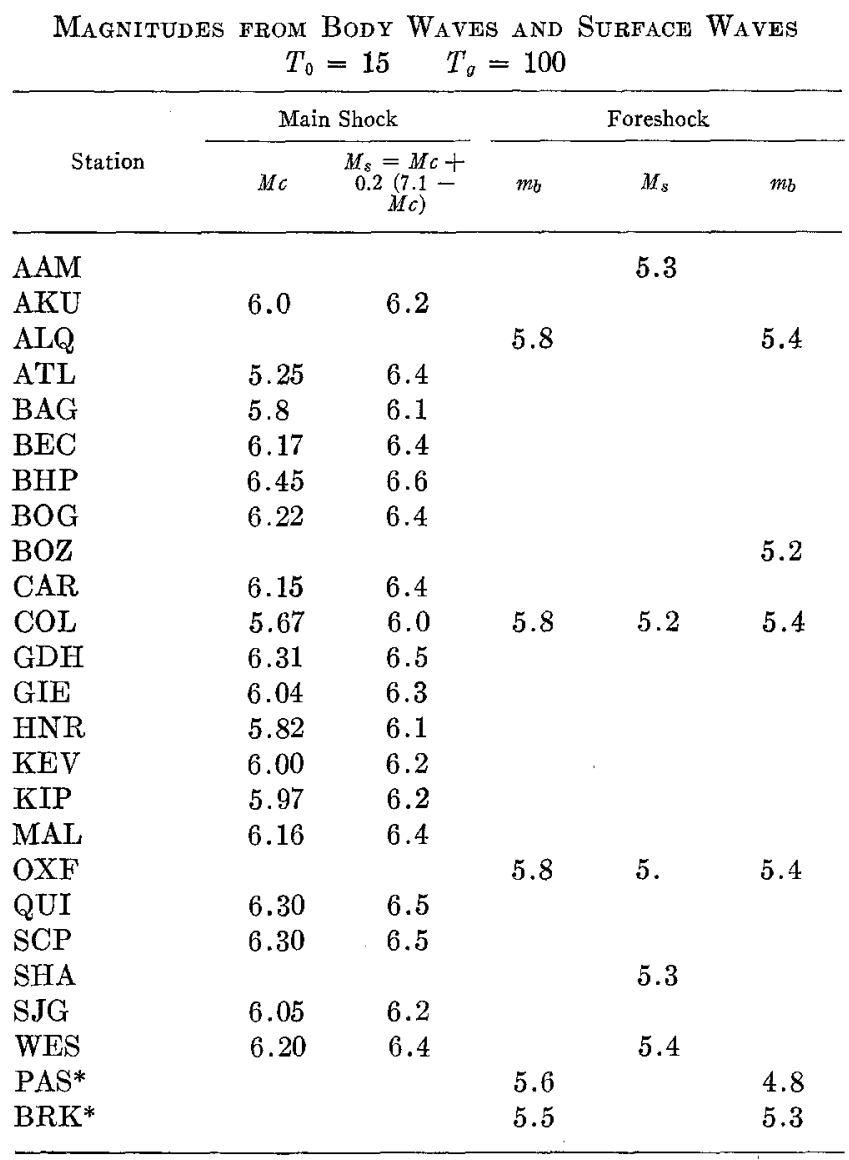

continental shelf may also partially explain the low amplitude at COL, since a major part of its energy is guided within the crust. Taking the radiation pattern into account, we would give more weight to the magnitudes derived from stations near the maximum of the lobes (See next section).

One interesting fact demonstrated by the foreshock and mainshock magnitudes from body waves and surface waves is that for the foreshock $M_{b} \cong M_{s}=5.4$ while for the mainshock $M_{s}-M_{b} \cong 6.4-5.8=0.6$. The empirical equation relating $M_{b}$ and $M_{\varepsilon}$,

$$
M_{b}=0.63 M_{8}+2.5
$$


(Gutenberg and Richter, 1956) would yield $M_{b}=5.9$ and 6.5 for the foreshock and mainshock respectively from $M_{s}$ 's and $M_{s}=4.62$ and 5.25 for the foreshock and mainshock respectively from $M_{b}$ 's. In other words $M_{s}^{\prime}$ 's for both the foreshock and the aftershock are higher than "average" earthquakes and the anomaly is greater for the mainshock than for the foreshock. Higher excitation of surface waves

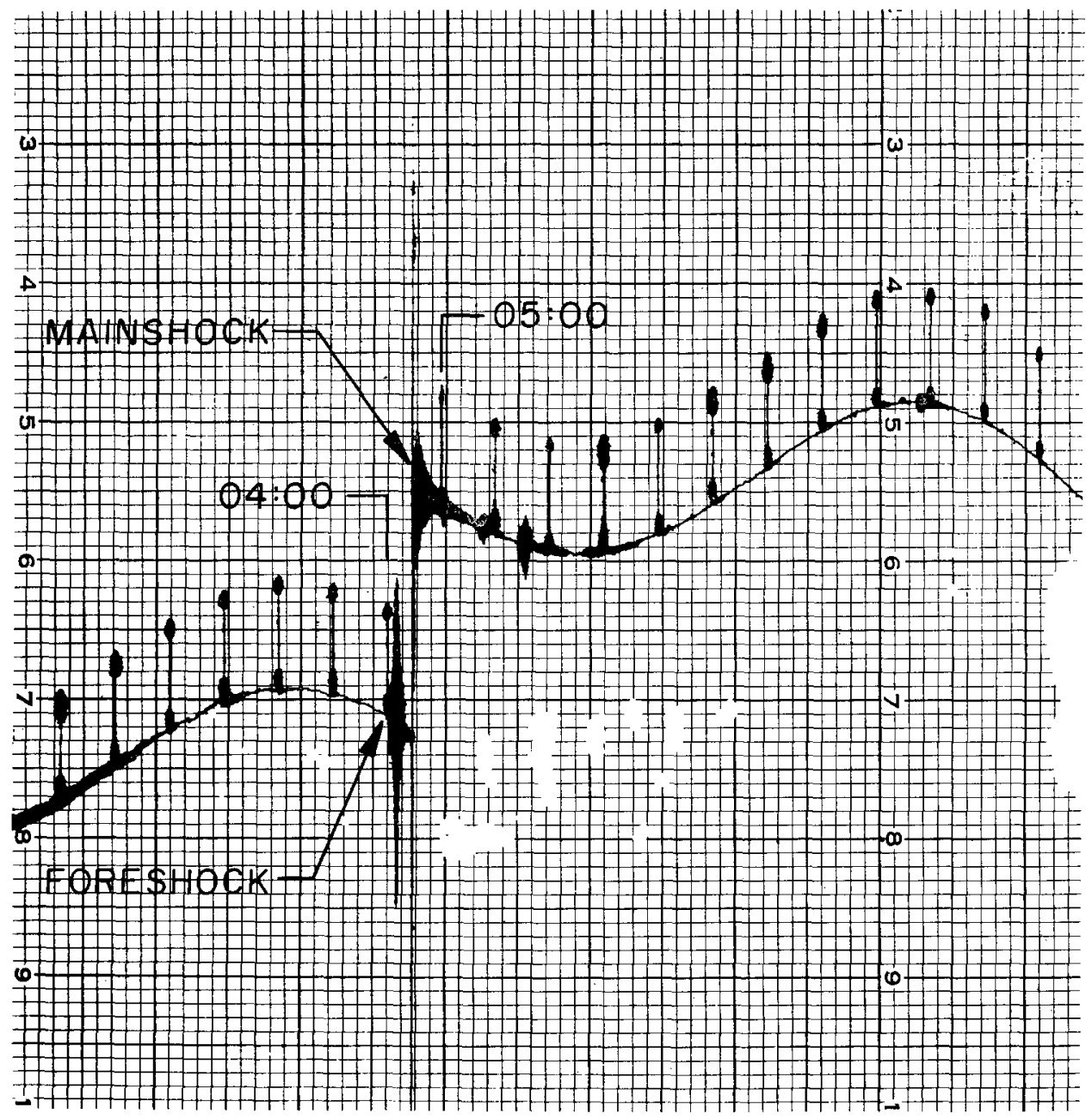

Fig. 1. Tiltmeter recording at Isabella, California. Notice the large offset associated with the main shock.

is recorded elsewhere for California earthquakes (Brune and Allen, 1967), however not in the proportion shown by the mainshock. This discrepancy could be due to the relatively high excitation at the long-period end of the spectrum, as $M_{b}$ is determined by the 1 second energy while $M_{s}$ is determined by 20 second energy. The high excitation of long-period content evidently extends to the DC end of the spectrum (Figure 1); there is no apparent DC offset associated with the foreshock while for the mainshock the offset is of the order of $10^{-7}$. 


\section{Surface-Wave Radiation Pattern and the Source Mechanism}

Surface waves from the mainshock (especially $R_{1}$ and $G_{1}$ ) are excellently recorded at many U.S. stations, Canadian stations, and stations across the Pacific.

Source mechanism studies based on surface waves were mainly a study of average features of the source. Ben-Menahem (1961) studied the phenomenon of propagating rupture. Brune (1961), Ben-Menahem and Harkrider (1964) and Wu and BenMenahem (1965) studied the radiation pattern of an earthquake source at different frequencies. The previous studies were made on large earthquakes (1960 Chilean, 1962 Iran, and so on), Parkfield earthquake is one that is relatively small and yet generated enough surface waves to be recorded at long distances. Attention will be directed mainly at periods less than 50 seconds. We will attempt to correlate the surface wave results with strong motion and seismoscope data.

The long-period data records used in this work are from WWNSS and Canadian stations; they are listed in Table 2 together with other pertinent data. These records are digitized on an Oscar digitizer with irregular increment, and later interpolated at the desired interval.

The digitized records are subjected to

(1) Trend removing

(2) Fourier Analysis

(3) Correction for attenuation

(4) Correction for distance effect on a sphere.

The attenuation coefficients for the period range 10-50 have been worked out by Trygvasson (1965) using explosion data. All the amplitude spectra were normalized to a distance of $5000 \mathrm{~km}$, this is an intermediate distance for the data and most of the data is close to this range. This was done in order to minimize the possible error caused by using coefficients not proper to the specific propagation path in question. Since there is no Airy phase in the period range under consideration, the combined attenuation and distance correction for surface waves can be written as

$$
A_{0}(\omega)=\frac{A(\omega) e^{\delta(\omega)(d-5000)} \sqrt{\sin \Delta \cdot \Delta}}{\sqrt{\sin 45^{\circ} \cdot \pi / 4}}
$$

where

$$
\begin{aligned}
\omega & =\text { frequency } \\
A(\omega) & =\text { uncorrected spectrum } \\
\delta(\omega) & =\text { attenuation coefficient } \\
d & =\text { epicentral distance in } \mathrm{km} \\
\Delta & =\text { epicentral distance in radians } \\
A_{0}(\omega) & =\text { corrected spectrum. }
\end{aligned}
$$


In addition to the reason mentioned above for not equalizing the amplitude to the epicenter, it is also clear from this formula that the approximate distance correction factor on a sphere $\sqrt{\sin \Delta \cdot \Delta}$, which is valid for $\Delta>\pi / 12$ would nullify the spectrum.

Amplitudes at various periods are read off and plotted on a polar graphpaper with the epicenter at the origin, care being taken to avoid the minima in the spectra, or to smooth over them. This procedure is performed under the assumption that the

TABLE 2

WWNSS and Canadian Station Data

\begin{tabular}{|c|c|c|c|}
\hline & $\Delta(\mathrm{deg})$ & $\Delta(\mathrm{km})$ & Azimuth (deg) \\
\hline AMM & 29.1 & 3239 & 66.0 \\
\hline $\mathrm{AFI}$ & 69.3 & 7710 & 234.1 \\
\hline AKU & 62.6 & 6955 & 27.1 \\
\hline $\mathrm{ALQ}$ & 11.5 & 1278 & 90.7 \\
\hline ATL & 29.7 & 3307 & 84.1 \\
\hline $\mathrm{BAG}$ & 102.2 & 11373 & 300.7 \\
\hline $\mathrm{BEC}$ & 45.8 & 5093 & 77.5 \\
\hline BHP & 45.9 & 5104 & 115.6 \\
\hline $\mathrm{BOG}$ & 52.8 & 5876 & 114.5 \\
\hline $\mathrm{BOZ}$ & 12.1 & 1345 & 33.3 \\
\hline CAR & 54.5 & 6064 & 103.8 \\
\hline $\mathrm{COL}$ & 33.3 & 3700 & 339.1 \\
\hline GDH & 48.8 & 5421 & 25.8 \\
\hline GIE & 46.0 & 5123 & 135.7 \\
\hline HNR & 87.1 & 9695 & 256.3 \\
\hline $\mathrm{KEV}$ & 72.0 & 7995 & 11.3 \\
\hline KIP & 35.7 & 3972 & 256.5 \\
\hline MAL & 86.5 & 9614 & 46.3 \\
\hline $\mathrm{OXF}$ & 25.4 & 2823 & 84.0 \\
\hline QUI & 52.7 & 5868 & 122.8 \\
\hline RIV & 107.7 & 11984 & 240.9 \\
\hline SCP & 33.5 & 3729 & 68.5 \\
\hline SHA & 26.4 & 2933 & 83.1 \\
\hline STG & 50.9 & 5660 & 95.3 \\
\hline WES & 38.3 & 4252 & 64.9 \\
\hline $\mathrm{BLC}$ & 32.2 & 3576 & 19.9 \\
\hline FFC & 22.9 & 2546 & 28.3 \\
\hline $\mathrm{FST}$ & 18.8 & 2090 & 253.4 \\
\hline $\mathrm{FBC}$ & 42.0 & 4662 & 31.7 \\
\hline RES & 40.9 & 4540 & 10.1 \\
\hline
\end{tabular}

minima in the spectra are effects of the fault being a propagating rupture, or closely spaced multiple events, or that along the path the waves are selectively filtered. In avoiding or smoothing over the spectral minima, the plot of the radiation pattern loses information about the above-mentioned features of the source, in exchange for the reduction of the scattering of data. Looking at the equalized spectra, (Figure 6), it is obvious that in the period range of 50-30 seconds, smoothing does not have much effect on the amplitudes, and that in the shorter periods smoothing becomes necessary. It is well known that if the propagating source or multiple events are an 
accumulation of body force equivalents of the same type, the amplitude will be modified by $\sin x / x, \cos x$ or $\sin x$ type of functions; therefore, smoothing enables us to reduce the data scattering and recover the body force equivalents.

The plotted radiation patterns are shown in Figure 2. For periods shorter than 25 seconds the scattering of data is considerable; this results obviously from the fact that at periods less than 25 seconds the wavelengths $(\sim 90 \mathrm{~km})$ are comparable to the fault length (approximately $40 \mathrm{~km}$ from surface evidence), and the higher order features of the source appear in the waves. At periods greater than 25 seconds, the radiation patterns of Rayleigh waves have the standard four-lobed structure. The data for Love waves are not as complete as those for Rayleigh waves; however, the radiation patterns do show a four-lobed structure with maxima and minima located

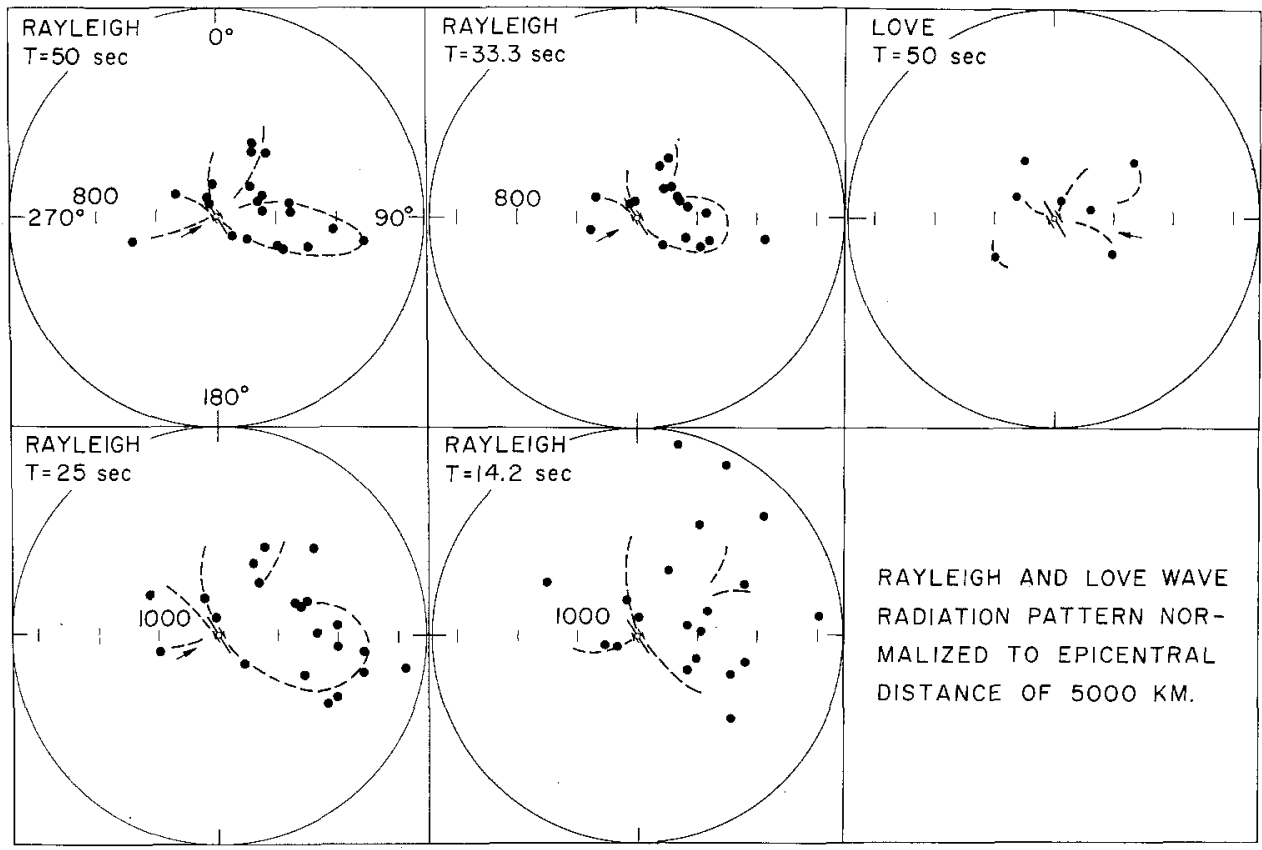

FIG. 2. Experimental radiation patterns.

at the minima and maxima, respectively, of the Rayleigh wave radiation patterns. This is illustrated in Figure 3. At Riverview (RIV), Australia, rather prominent Love waves are found on the horizontal components, while virtually no Rayleigh waves appear on the vertical or the horizontal component. At KEVO (KEV), Norway, on the contrary, the Rayleigh waves are prominent while Love waves are very small. Together with the observation that at College (COL), Alaska, the Love waves are fairly large we conclude that the source, for wavelengths long enough, can be represented adequately as a double couple. The orientation of the double couple can be anchored quite accurately by using the RIV and KEV records; the direction of the faulting, however, is not immediately clear from the present data. The field evidence unequivocally yields N $33 \mathrm{~W}$ as the fault strike. The $P$-wave fault plane solution (McEvilly, 1966) gives the same result; in addition the solution 

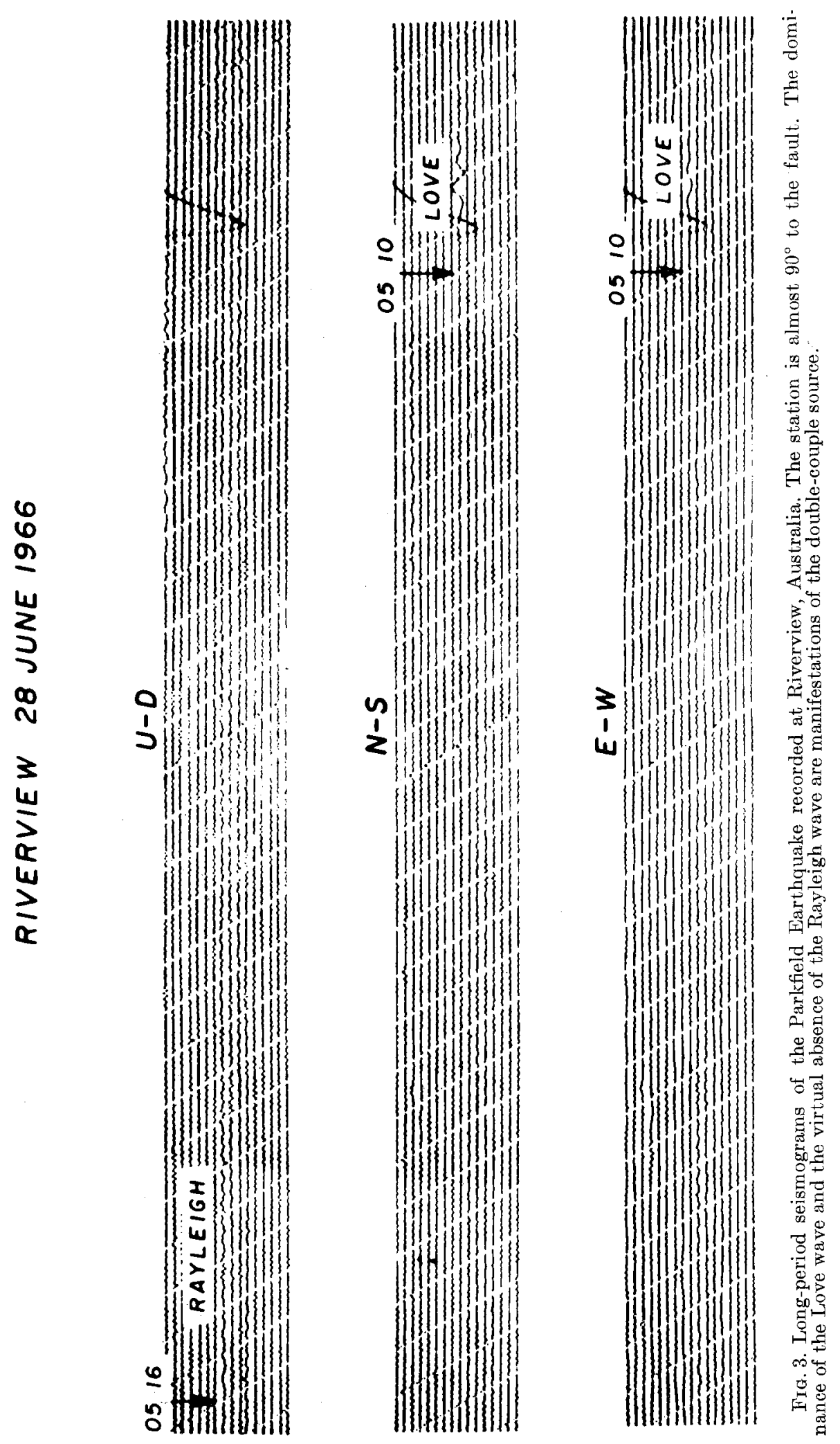
indicates that the fault plane is essentially vertical and has a $\lambda$ of $20^{\circ}$ (i.e., a strikeslip fault with a small vertical component).

The surface-wave data agree with the conclusions drawn from the fault plane solution. Figure 4 presents the theoretical surface-wave radiation patterns for source depths of (1) 4.5 and (2) $8.6 \mathrm{~km}$ and for each depth two sets of $\delta$ and $\lambda$ 's: (a) $\delta=90^{\circ} \lambda=0^{\circ}$ and (b) $\delta=90^{\circ} \lambda=20^{\circ}$ (as defined in Ben-Menahem and Harkrider, 1964). Love wave patterns for all cases and Rayleigh wave patterns for cases 1 a and 2a are rather insensitive to the depth and the periods. The Rayleigh wave patterns for cases $1 b$ and $2 b$ at 50 seconds are essentially four-leaf clover shaped, but at 25 seconds the Rayleigh wave pattern symmetry becomes two-fold, with medium intensity radiation $90^{\circ}$ from the direction of the strike of the fault; this phenomenon is more pronounced in the $8.6 \mathrm{~km}$ case than in the $4.5 \mathrm{~km}$ case. Seismograms at Seven Falls, Canada, (SFA) (which is approximately $90^{\circ}$ from the strike direction) have quite large 20 second Rayleigh waves, with very little 50 second energy. Comparing SFA and $\mathrm{KEV}$ records it seems possible that the apparent fault depth is about $8 \mathrm{~km}$.

So far we have discussed the equivalent force aspect of the source. Contained in the amplitude and phase spectra is information about the temporal and/or spatial behaviour of the source. If the source can be represented by a smoothly moving rupture then both the fault length and the velocity of propagation can be found (Ben-Menahem, 1961). The initial phase is not very easy to obtain without the precise phase velocity for each individual path. On the other hand, the amplitude spectra will be modified by a

$$
\frac{\sin \frac{\pi b}{\lambda}\left(\frac{c}{v}-\cos \theta\right)}{\frac{\pi b}{\lambda}\left(\frac{c}{v}-\cos \theta\right)}
$$

factor, where $b=$ fault length, $\lambda=$ wavelength, $c=$ phase velocity, $v=$ propagation velocity and $\theta=$ azimuthal angle with epicenter as origin and $\theta=0$ in the direction of the fault. For a fixed set of $b$ and $v$, minima in spectra, corresponding to $b / \lambda(c / v-$ $\cos \theta$ ) $=n, n=1,2 \cdots$, would occur at different $\lambda$ (i.e. period $T$ for given $c$ ) for different $\theta$ (see $\mathrm{Wu}$ and Ben-Menahem, 1965). By matching the theoretical curves with the observed spectra at different azimuthal angles we can find a best-fit faultlength and rupture propagating velocity for the earthquake (see, for example, Ben-Menahem and Toksoz, 1963). In the present case, at CAR, say, $\theta=45^{\circ}$ and the destructive interference condition

$$
\frac{b}{\lambda}\left(\frac{c}{v}-\cos \theta\right)=1
$$

yields

$$
\frac{1}{v}-0.185=\frac{T_{z}}{b}
$$




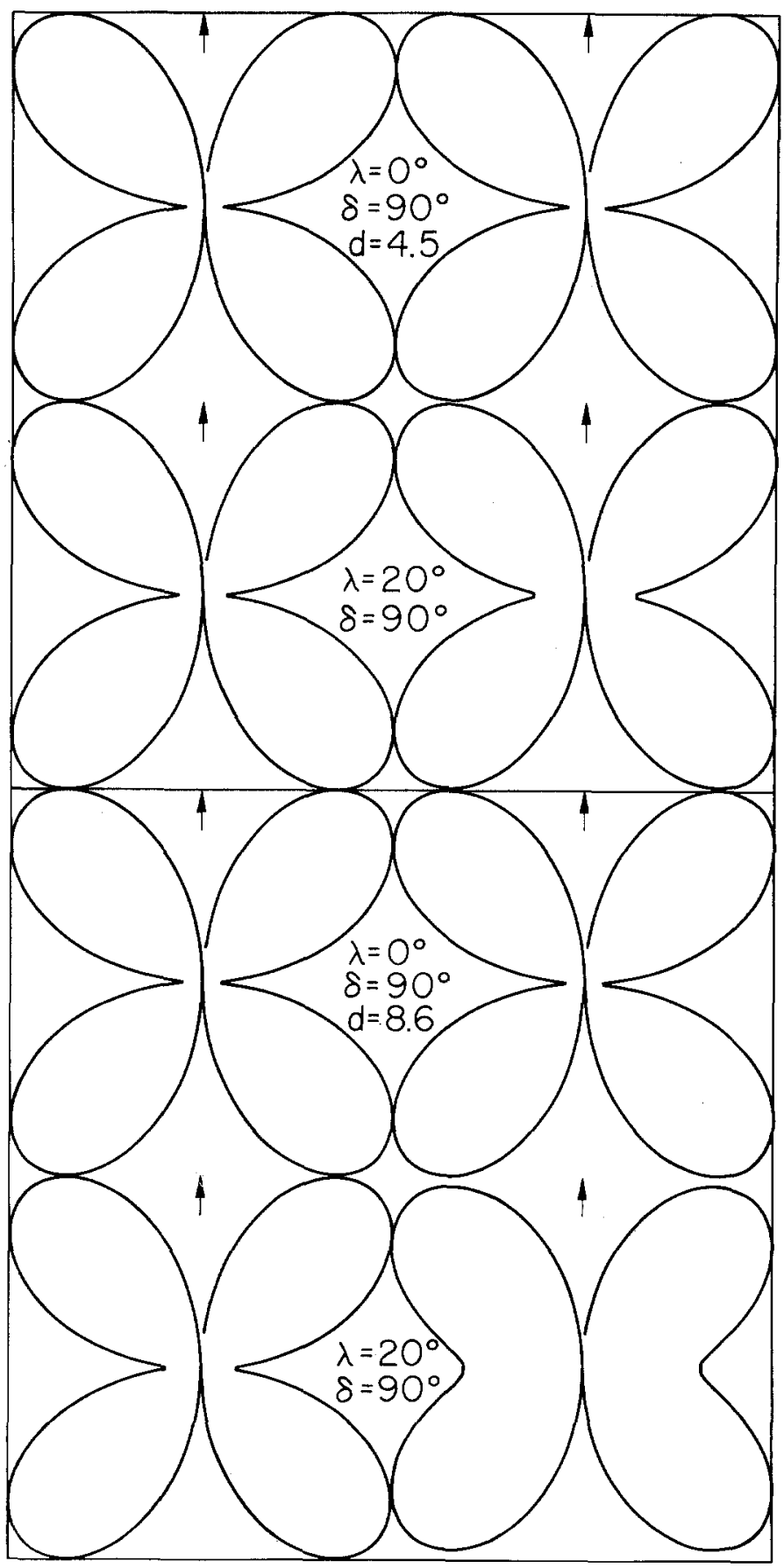

FIG. 4. Theoretical Rayleigh wave radiation pattern from a vertical strike slip fault with $20^{\circ}$ rake at two depths and two periods at left $T=50$ seconds and at right $T=25$ seconds. 
$c$ being taken to be $3.8 \mathrm{~km} / \mathrm{sec} T_{z}=$ the period at which spectral minimum occurs. The amplitude spectrum gives $T_{z}=28 \mathrm{sec}$; with $b$ having $40 \mathrm{~km}$ as upper bound.

$$
v_{\max }=1.3 \mathrm{~km} / \mathrm{sec} \text {. }
$$

Such a rupture velocity is found to be lower than the values predicted by experiments or previous rupture velocity determinations (Ben-Menahem and Toksoz, 1961). An alternative source mechanism in view of the present data as well as strong motion and seismoscope data (see next section) is that the earthquake was an accumulation of closely spaced multiple events.

In the most simple case, two events with the same time function, with a lag of $\Delta t$ between them, and an amplitude factor of $\mathbf{a}$ for the second pulse expressed in time domain as

$$
g(t)=f(t)+\mathbf{a} f(t-\Delta t)
$$

The spectrum of $g(t)$ is

$$
G(\omega)=F(\omega)\left\{1+\mathbf{a} e^{-i \omega \Delta t}\right\}
$$

where $G(\omega)$ and $F(\omega)$ are the Fourier transforms of $g(t)$ and $f(t)$ respectively. This last equation shows that $F(\omega)$ will be modified by a function

$$
1+\mathbf{a} e^{-i \omega \Delta t}
$$

The amplitude and phase of this function are plotted in Figure 5. These graphs show that amplitude minima occur at $\omega \Delta t=n \pi$, and the amount of dip depends on the parameter a. In addition, the phase has jumps at $\omega t=n \omega$, the amount depends again on a. The situation starts to multiply in complexity if there are more than two events in the series and the successive source time functions are even slightly different; then, the dips would not occur at regular intervals, nor would the amplitude have a regularly repeating pattern.

In Figure 6 we have shown the equalized spectra at different azimuthal angles. At most of the stations there is a distinct spectral minimum between 20 and 30 seconds. The four southeastern stations CAR, BHP, BOG and QUI differ only slightly in azimuthal angle with respect to the source and have very closely matching first minima (with longest period). Continental station ATL shows a very small and doubtful trough at 27.5 seconds, on the other hand LON spectrum has a very clear one at 29 seconds. It is interesting to note that at COL the spectrum has a minimum 22 second coinciding with the Berkeley Love wave spectral minimum reported by Filson and McEvilly (1967); Berkeley and COL differ in epicentral distance but have nearly the same azimuth with respect to the source. BAG and HNR are very distant stations; the spectra at both these stations show a minimum at 22 second; BAG spectrum amplitude has irregularities at longer periods, but due to the low power at the period range the significance can probably be discredited. These periods are also plotted in the lower portion of Figure 6 as a function of the azimuthal 
angle $\theta$. The periods group around 26 seconds. Assuming this to be the first maximum i.e., $\Delta t=T_{z} / 2=13$ seconds. Thus, it is possible that two of the larger source pulses accur at an interval of 13 seconds. There are troughs in the spectra at shorter periods, near thirteen seconds; they intersperse with so many other
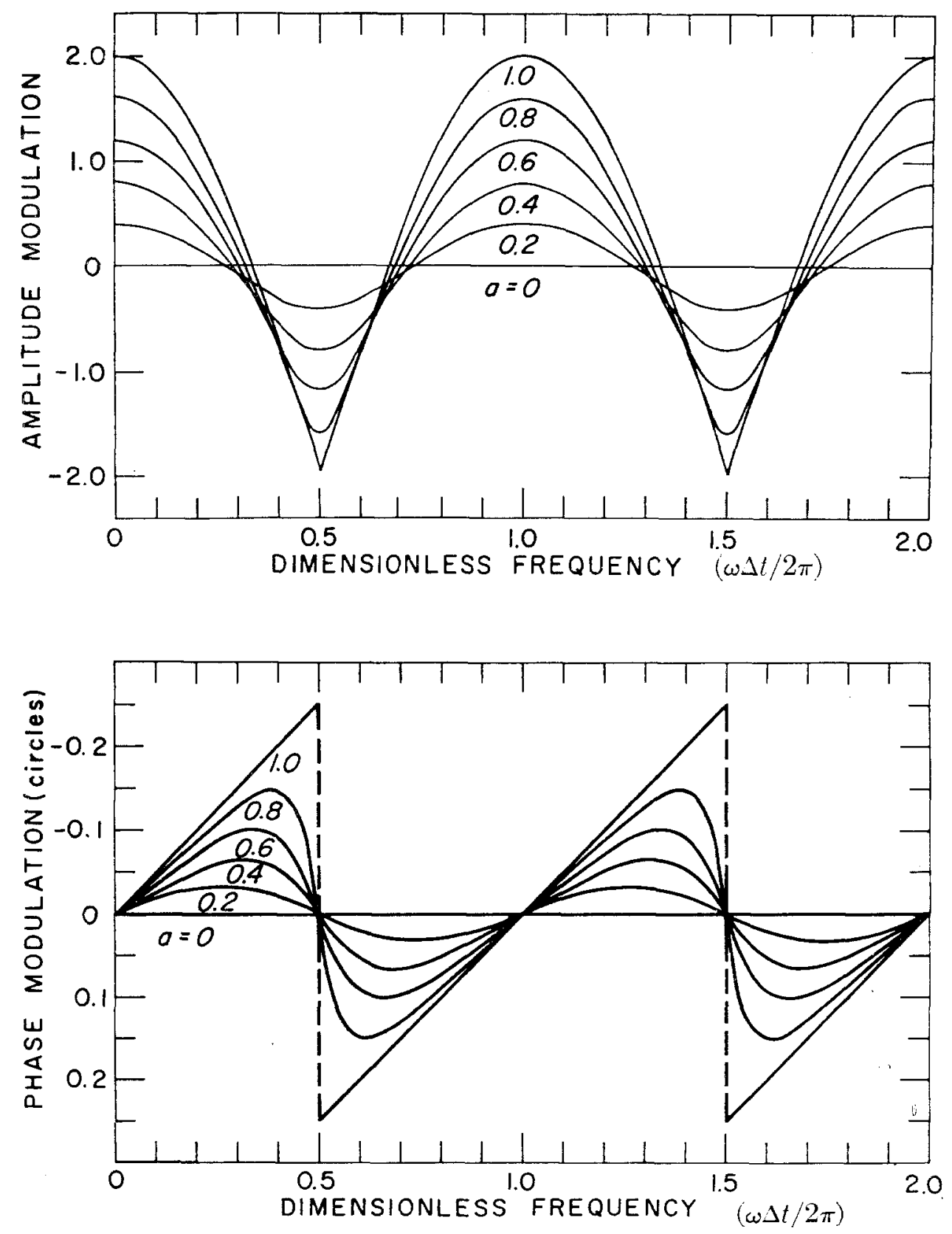

Fra. 5. Amplitude and phase of the function $1+a e^{-i \omega_{\Delta t}}$. 
troughs that could have been caused by crustal boundary reflections, local wave guides and so on; it ceases to be clear as to their identities.

By using the first order interference gap in the spectra we cannot distinguish whether the source is a multiple event or a smoothly and continuously moving rupture, with the constraints of fault length and phase velocity imposed. However,

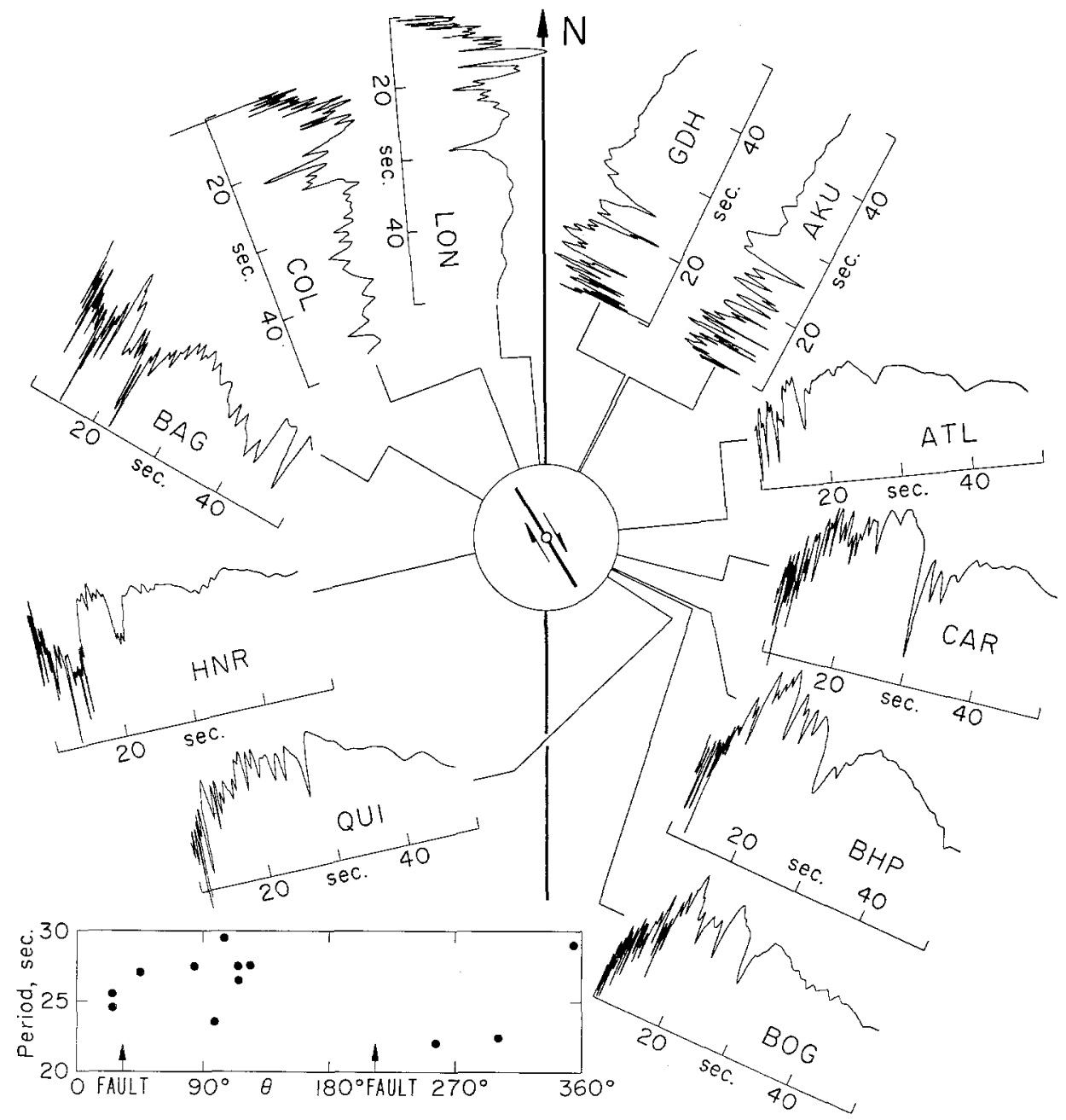

FIG. 6. Rayleigh wave amplitude spectra at stations around the source showing spectral minima. At lower left corner is a plot of the periods at which the first minima are located as a function of azimuthal angle.

it can be easily seen from the interference condition

$$
\frac{b}{\lambda}\left\{\frac{c}{v}-\cos \theta\right\}=1
$$

that in order to minimize the influence of $\cos \theta$, such that the placement of the gaps has the variance allowed by the data (Figure 6), we would have to make $c / v \gg 1$; 
with $c=4 \mathrm{~km} / \mathrm{sec}, v$ has to be less than $1 \mathrm{~km} / \mathrm{sec}$. This rupture velocity is too small compared to the laboratory rupture velocity.

\section{Parkfield Earthquake as Multiple Events}

Surface wave data suggested that the Parkfield earthquake could have been a series of events closely spaced in time with two of the larger events separated by 13 seconds. This picture is supported by several other lines of evidence (see Figure 7 ).

(1) Seismoscope data. (Figure 7a) the seismoscope array installed in the immediate vicinity of the ground breakage was useful in deciphering the orbital motion of the waves. Even though the records are cluttered and time sequence can not be established on them, it is evident that the waves came from more than one direction, at different times. The orbits are more or less ellipsoidal with diverse ellipticity; at one instant the major axis seems to be perpendicular to the direction of the fault, at next it is diagonal to the fault or parallel to the fault. It is possible that these orbital motions are controlled, to some degree, by the local geology; one main factor influencing them was probably the position of the source at that moment as depicted in Figure 7 d. Needless to say, the amplitude of the motions depend on the distance from the source to the receiver and the strength of the source.

(2) Strong-motion seismograms. Because of the lack of absolute time on the seismograms they cannot be used to obtain the arrival times of the waves recorded. The wave shape, however, lends us clues as to the possible source-time function. Since the free period of the pendulum is around 0.06 , the ground motions with periods much longer than 0.06 are recorded as accelerations; every pulse (Figure 7b) on the seismogram represents a "jerk" in the displacement. Some of the smaller pulses might be lateral or vertical reflections, but the larger ones must be associated with source motion. The apparent duration of the source from each seismogram is determined by the velocity at which the source travels and the attenuation of the waves; it is shown in the same set of data that beyond a distance of nine miles, the amplitude is reduced to noise level.

(3) Long-period and short-period teleseismic signals. Long-period body waves recorded in the distance range $10-35^{\circ}$ (examples in $7 \mathrm{c}$ ) are clear and consist of up to 5 distinct pulses at intervals of 4-7 seconds; they suggest the complex behaviour of the source. Unfortunately, $P$ waves in this distance range are usually complicated by arrivals associated with triplications in the travel-time curves (Johnson, 1967; Archambeau and Flinn, 1967). However, the present data indicate arrivals much later than those predicted by travel-time curves. In Figure 8 the time $T-T_{0}$, where $T_{0}$ is the time for the first $P$ of the later pulses are plotted. For $T-T_{0}<13$ seconds some of the pulses could be explained by triplications, the arrivals around 16 seconds are probably related to the source. The time readings are not accurate enough to warrant a detailed analysis.

The short-period records of the mainshock are rather dissimilar to those of the foreshock; an example is given in Figure 9. The dissimilarities reflect the differences in source behaviour, since the travel path is the same.

The idea of some major earthquakes being multiple shocks has been proposed by previous authors (Florensov and Solonenko, 1963, Gobi-Altai earthquake; Wyss and Brune, 1967, Great Alaskan earthquake of 1964 among others). They based their investigations on the $P$ waves. The Parkfield earthquake provided near field 
data to support the conclusion from teleseismic data. Intuitively, it is unlikely that a rupture would propagate at uniform velocity along a long fault; stick-slip fault is probably the rule for any earthquakes that involves long seismogenic faulting. Brace and Byerlee found analogous behaviour for rocks under high pressure.

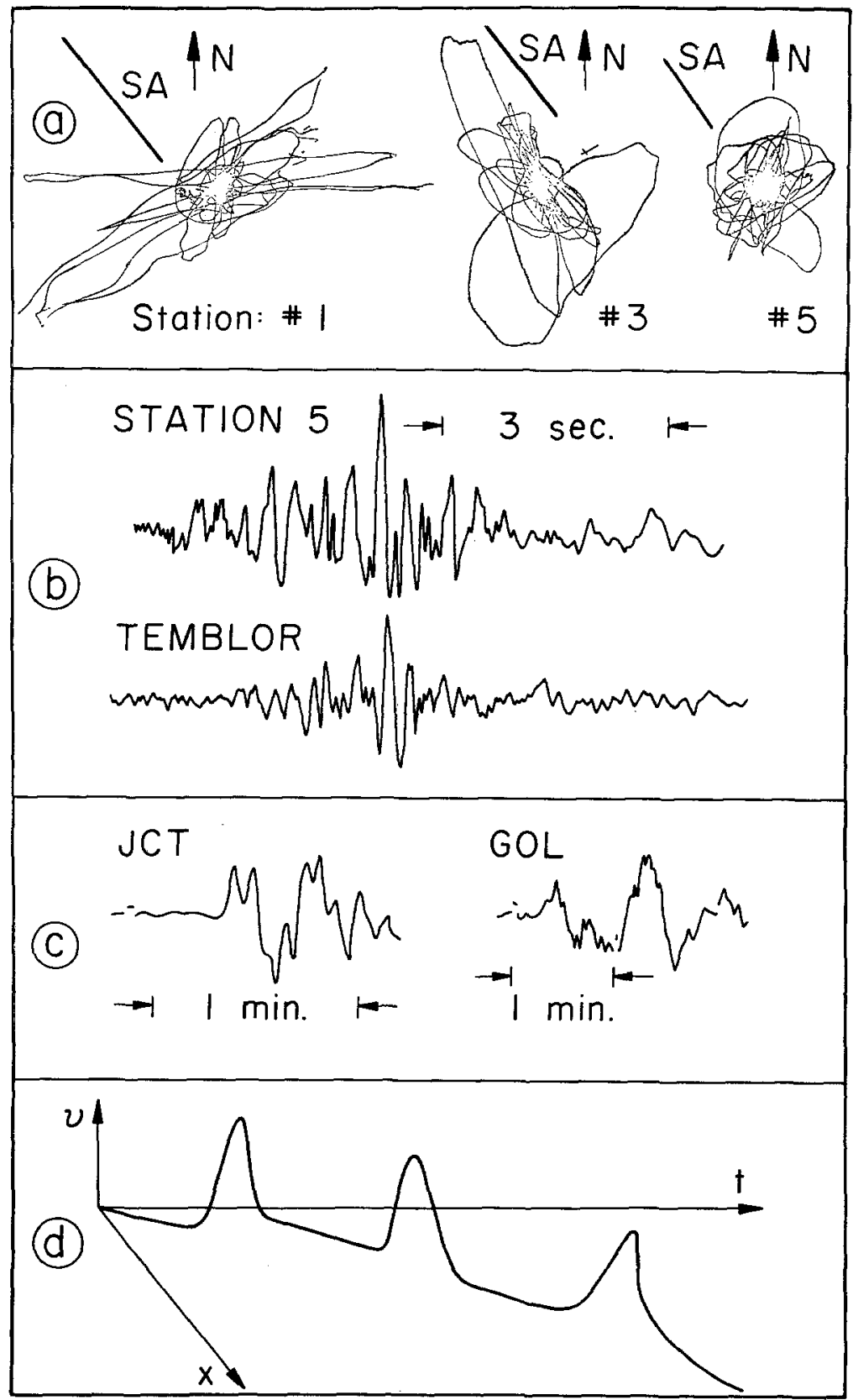

FIG. 7. Supporting evidence for the multiple shock hypothesis. (a) Seismoscope records, (b) strong-motion records, (c) teleseismic body waves, (d) schematic source temporal and spatial behavior. 
At present, without more direct evidence, we are proposing this as the most consistent way to explain the data. It would be desirable to set up strong-motion seismometer arrays with either radio controlled central triggering or absolute timing on the trace, along the fault and perpendicular to the fault, so that not only the sequence of events can be uncovered, but also the near-source behaviour of the waves.

\section{Entergy of Parkfield Earthquake}

The ordinary way of finding the energy of an earthquake from teleseismic data would certainly neglect the near-field energy; with the accelerometer data for the

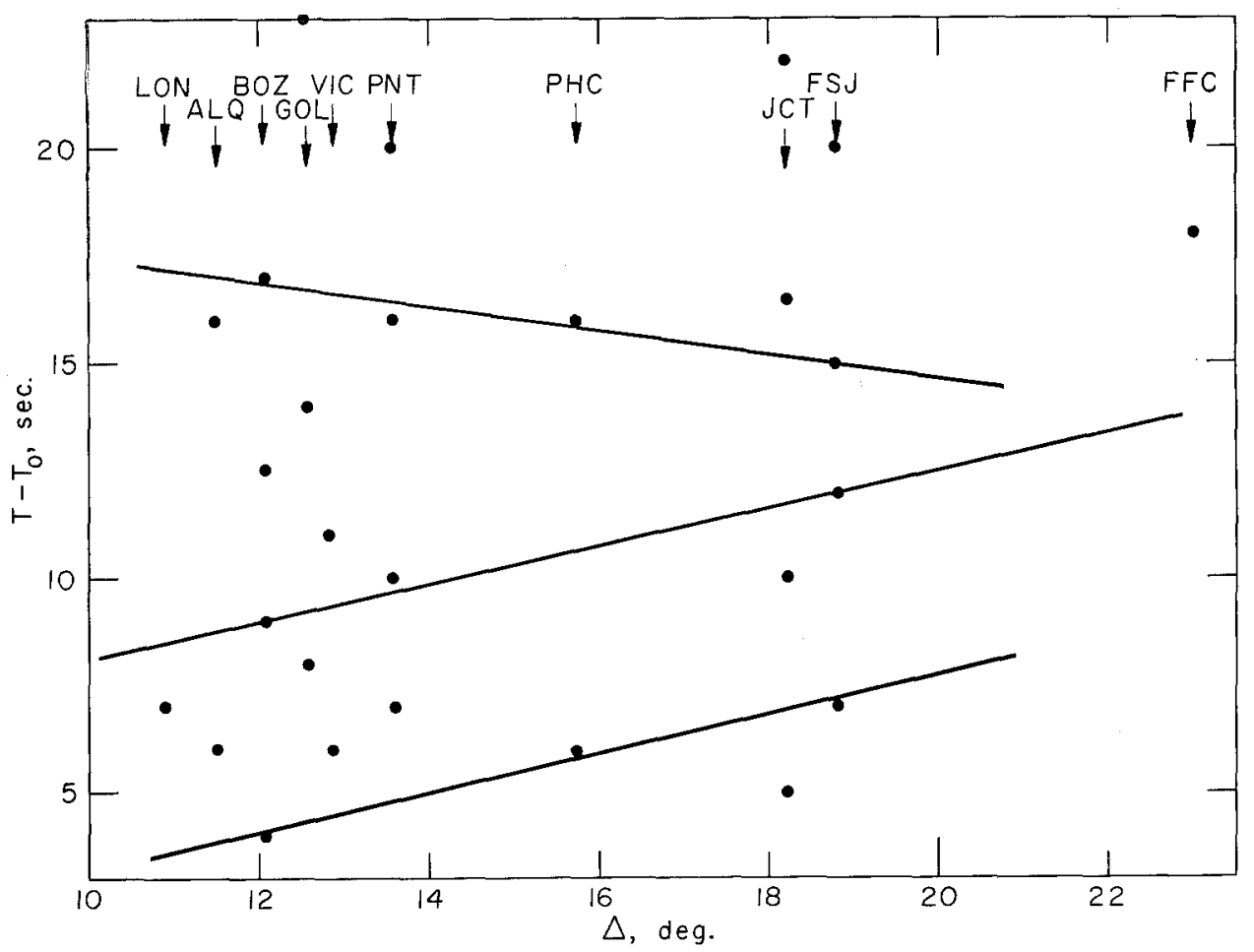

FIG. 8. Teleseismic arrivals. $T_{0}$ is the onset of the first motion.

Parkfield earthquake we are able to obtain a better estimate of the total energy. The response of the strong-motion seismometer to ground acceleration is essentially flat much beyond the pendulum period of around 0.06 seconds. The ground motions at shorter periods are registered as displacements. Since the main signals recorded have much longer periods than 0.06 seconds, we shall assume the response to be absolutely flat and integrate the seismograms directly to obtain velocity and use the formula

$$
\int_{0}^{T} \int_{A} \rho v\left\langle\frac{d t}{\partial t}\right\}^{2} d a d t
$$

to obtain the total energy of the source. A, the area of integration, has to be esti- 


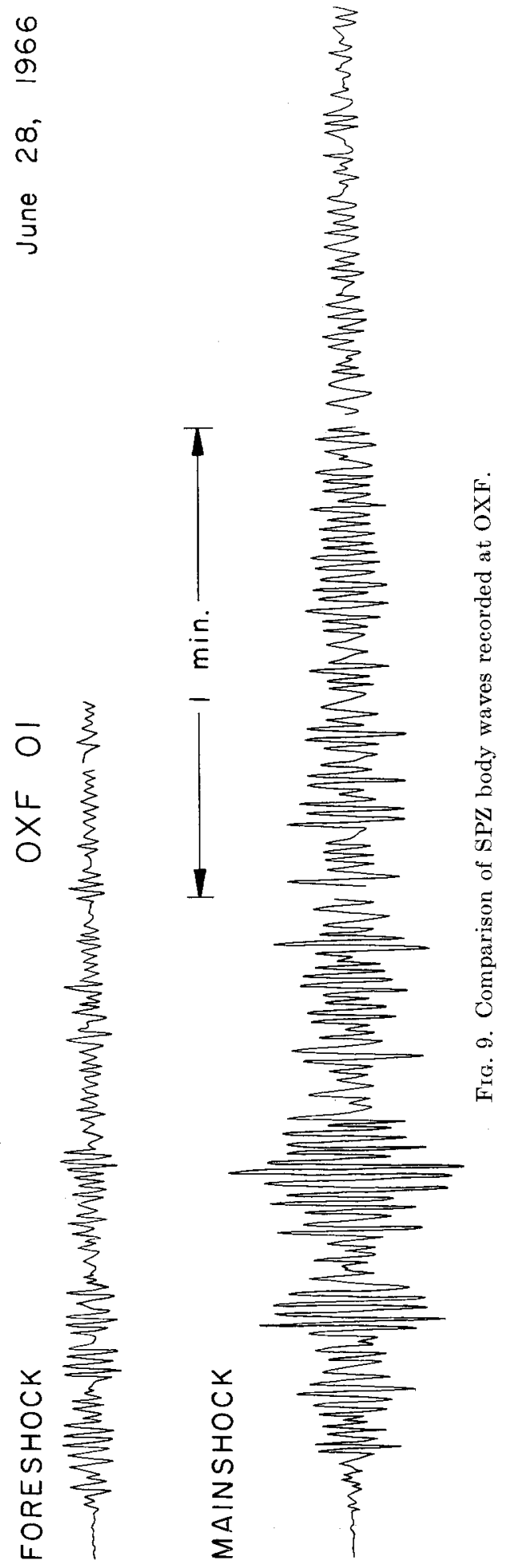


mated from the surface evidence, hypocentral information and aftershock locations. We shall use only the temblor station, which is located in granite, for our calculations. In doing so it is necessary to assume that the waves recorded at that station are common to all stations at that distance $(6.3 \mathrm{~km})$ away from the fault trace; this assumption is not unreasonable in view of the recordings at station 5 , the distances between the nearest fault trace and those two stations are nearly the same, but on different sides of the fault; the amplitudes at these two stations are comparable.

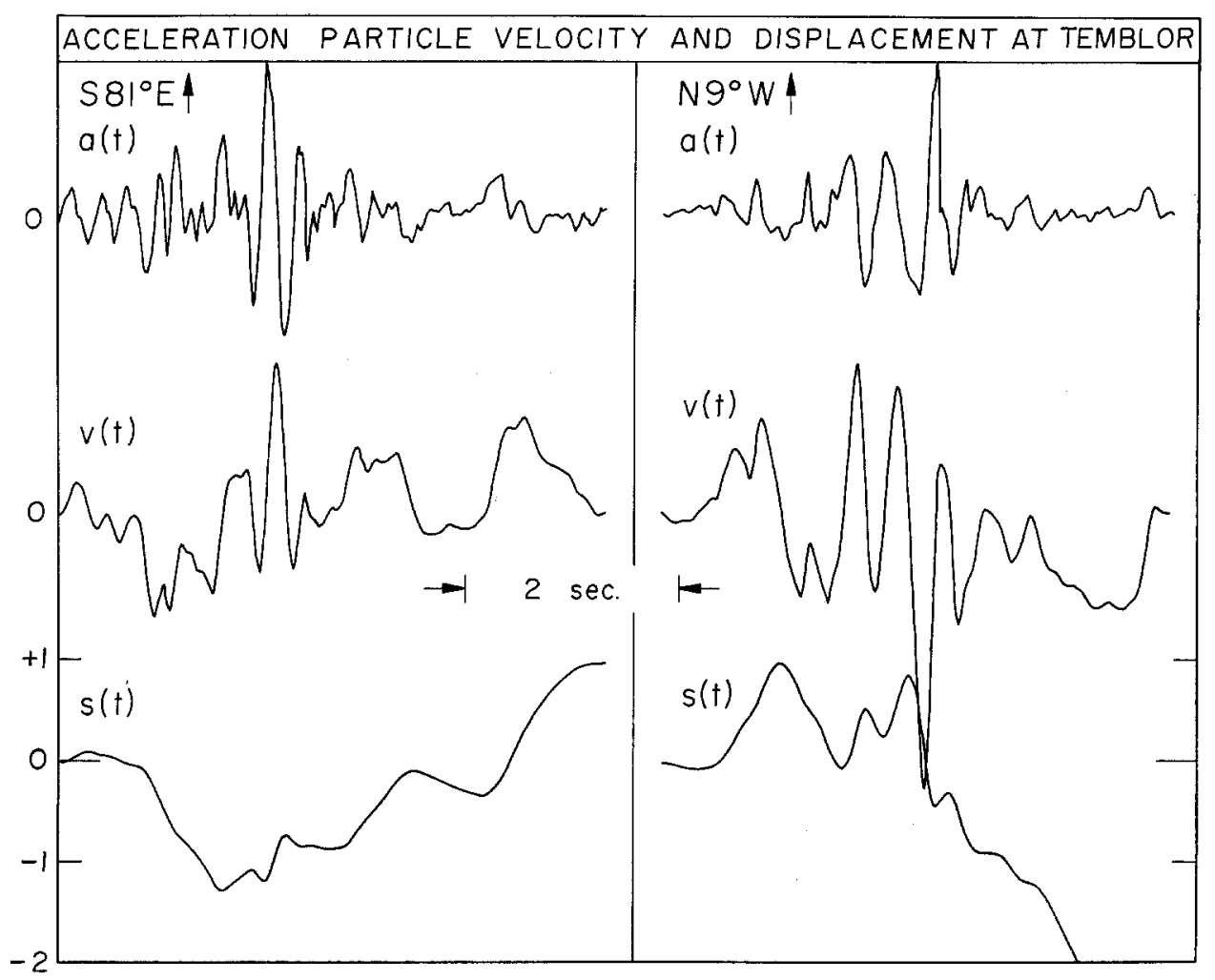

FIG. 10. Integration of strong-motion seismograms.

In integrating the accelerograms the initial conditions $a(t)=0$ and $v(t)=0$ were used. The accelerograms were digitized at very small but unequal intervals and the trapezoid rule is used for integration.

Assume

$$
\begin{aligned}
& \rho=2.7 \mathrm{gm} / \mathrm{cm}^{3} \\
& v=3 \times 10^{5} \mathrm{~cm} / \mathrm{sec} \\
& A=3.5 \times .5 \times 10^{12} \mathrm{~cm}^{2}
\end{aligned}
$$


and, for the accelerograms, the integral

$$
\int\left\{\frac{d s^{2}}{d t}\right\} d t=3.34 \times 10^{2} \mathrm{~cm}^{2} / \mathrm{sec}
$$

the energy comes out to be

$$
1 \times 10^{21} \text { ergs. }
$$

Using Gutenberg and Richter's formula

$$
\log E=11.8+1.5 M
$$

the energy is $E_{b}=3 \times 10^{20}$ ergs from $M_{b}=5.8$ and $E_{s}=3.5 \times 10^{21}$ ergs from $M_{s}=6.5$.

The estimation of energy based on strong-motion records is subject to errors in choosing the surface of integration and assuming the amplitude variations. The latter assumption is probably valid as we pointed out before and a small change in the area of integration will not change the final magnitude of energy very much. However, if there is significant energy dissipation in the region between the station and the source the energy will be higher.

The above contradiction of $E_{b}$ and $E_{s}$ can be accounted for by the observation that the earthquake is composed of several events in an interval of more than 10 seconds; $M_{b}$ is obtained from the first few cycles of body waves within a few seconds while 20 second waves reflect the effect of the long source-time function; thus $E_{b}$ would be an underestimate and $E_{s}$ might be an overestimate if the excitation function peaks at 20 seconds while low at other frequencies.

Assume the fault occurs in a pre-stress medium, then according to Keylis-Borok (1957), we can calculate the pre-stress involved using the energy obtained above.

$$
p^{2}=\frac{\mu E}{S 3 / 2 R_{S}}
$$

$$
\text { with } \begin{aligned}
\mu & =\text { rigidity }=3 \times 10^{11} \\
E & =10^{21} \mathrm{erg} \\
S & =1.75 \times 10^{12} \mathrm{~cm}^{2} \\
R_{S} & =1
\end{aligned}
$$

$p$ is found to be of the order of 10 bars. Inherent in the solution is the condition that $p=0$ on the fault surface after the earthquake.

In the case where pre-stress drops from 1 to $\gamma$ on the fault during the process then the solution can be modified by a factor of $\left(1-\gamma^{2}\right) / 2$ (Burridge and Knopoff, 1966), 
since during the faulting the stress drops from 1 to $\gamma$ and the displacement increases from 0 to $(1-\gamma)$ continuously.

$$
\delta w \sim \int_{\gamma}^{1} \gamma d \gamma=\frac{1}{2}\left(1-\gamma^{2}\right)
$$

Thus if $\gamma=0.9, p \sim 33$ bars.

\section{ConcLusion}

We have shown that the Parkfield earthquake is characterized by (1) the divergence between the body-wave and surface-wave magnitudes as contrasted by the convergence of the magnitudes for the foreshock, (2) the radiation patterns of surface waves are those corresponding to a double couple at a depth of about $8.6 \mathrm{~km},(3)$ the source can probably be represented by an accumulation of sources separated in time and space, (4) the total energy emitted is of the order $10^{21}$ ergs.

These conclusions were drawn mainly from teleseismic records. Many of the details of source could have been obliterated by the propagation effects of the waves. To study the source mechanism it is perhaps desirable to establish a two-dimensional array of strong-motion seismometers in a well-defined earthquake source region with radio time code and centralized triggering. Such an array would enable us to study not only the periods and the amplitudes of the strong-motion waves, but also the temporal and spatial behavior of the faulting process. This information is important in knowing the exact nature of earthquakes.

\section{ACKNOWLEDGMENTS}

The author wishes to thank Professor James N. Brune for stimulating discussions.

This research was supported by the Air Force Office of Scientific Research, Office of Aerospace Research, United States Air Force, under AFOSR contract AF-49(638)-1337.

\section{REFERENCES}

Archambeau, C. B. and E. A. Flinn (1967). Perturbation methods for the inversion of body wave travel-time data, $J$. Geophys. Res. (in press).

Bath, M. (1952). Earthquake magnitude determination from the vertical component of surface waves, Bull. Seism. Soc. Am. 35, 81-91.

Ben-Menahem, A. (1961). Radiation of seismie surface waves from finite moving sources, Bull. Seism. Soc. Am. 51, 401-435.

Ben-Menahem, A. and M. N. Toksoz (1963). Source mechanism from spectra of long-period seismic surface waves. 3. The Alaska earthquake of July 10, 1958, Bull. Seism. Soc. Am. 53, 905-919.

Ben-Menahem, A. and D. G. Harkrider (1964). Radiation patterns of seismic surface waves from buried dipolar point sources in a flat stratified earth, J. Geophys. Res. 69, 2605-2620.

Brune, J. N. (1961). Radiation pattern of Rayleigh waves from the southeast Alaska earthquake of July 10, 1958, Publ. of the Dominion Observatory, 24, 1-11.

Brune, J. N. and C. R. Allen (1967). A low-stress-drop, low-magnitude earthquake with surface faulting: The Imperial, California earthquake of March 4, 1966, Bull. Seism. Soc. Am. 57, 501-514.

Burridge, R. and L. Knopoff (1966). The effect of initial stress or residual stress on elastic energy calculations, Bull. Seism. Soc. Am. 56, 421-424.

Filson, J. and T. V. MeEvilly (1967). Love-wave spectra and the mechanism of the 1966 Parkfield sequence. Conference on Geologic problems of the San Andreas Fault System. Palo Alto, California, September 14-16. 
Florensov, N. A. and V. P. Solonenko (1963). The Gobi-Altai Earthquake, Isdatel'stvo Akademii Nauk SSR, Moscow, pp. 392. (English translation by Israel Program for Scientific Translations, (1965).

Gutenberg, B. (1945). Amplitude of surface waves and magnitudes of shallow earthquakes, Bull. Seism. Soc. Am. 35, 3-12.

Gutenberg, B. and C. F. Richter (1936). On Seismic Waves (Third Paper), Gerlands Beitr. 2. Geophysik, 47, 73-131.

Gutenberg, B. and C. F. Richter (1956). Magnitude and energy of earthquakes, Annali di Geofisica, 8, 1-15.

Johnson, L. R. (1967). Array measurements of $P$ velocities in the upper mantle, $J$. Geophys. Res. (in press).

Keylis-Borok, V. (1957). On estimation of the displacement in an earthquake source and of source dimensions, Annali di Geophys. 10, 205-214.

McEvilly, T. V. (1966). Preliminary seismic data, June-July, 1966, Bull. Seism. Soc. Am. 56, 967-971.

Richter, C. F. (1935). An instrumental earthquake magnitude scale, Bull. Seism. Soc. Am. 25, $1-32$.

Trygvasson, E. (1965). Dissipation of Rayleigh wave energy, J. Geophys. Res., 70, 1449-1455.

$\mathrm{Wu}, \mathrm{F}$. and A. Ben-Menahem (1965). Surface wave radiation pattern and source mechanism of the September 1, 1962 Iran Earthquake, J. Geophys. Res. 70, 3943-3949.

Wyss, M. and J. N. Brune (1967). The Alaska earthquake of 28 March 1964; A complex multiple structure, Bull. Seism. Soc. Am. 57, 1017-1023.

SEISMOLOGICAL LABORATORY

California Institute of Technology

Pasadena, California

(Division of Geologicat Sciences, Contribution No. 1493).

Manuscript received October 25, 1967. 